\title{
Non-IgE Medicated Food Allergy: Eosinophilic Esophagitis Update on the Pathogenesis, Clinical Features, and Management of Eosinophilic Esophagitis in Children
}

\author{
Matthew Greenhawt • Seema S. Aceves
}

Published online: 23 April 2014

(C) Springer Science + Business Media New York 2014

\begin{abstract}
Eosinophilic esophagitis (EoE) is a chronic allergic disorder of increasing worldwide prevalence. Diagnosis and management require upper gastrointestinal endoscopy with biopsy. Therapeutic strategies in children and adults include empiric and testing-based elimination diets as well as topical esophageal corticosteroids. The last 12 months have provided a number of insights including updated diagnostic/management guidelines, the role of PPI therapy, and an understanding that EoE disease mechanisms rely on basophils and mast cells in addition to eosinophils. Novel diagnostic techniques have been described and include the esophageal string test and a molecular gene array panel. In addition, new techniques for assessing esophageal rigidity have been reported. In this review, we summarize the most impactful findings in EoE over the last 12-15 months.
\end{abstract}

Keywords Eosinophil - Gastroesophageal reflux disease Proton pump inhibitor · Eosinophilic esophagitis · Food allergy $\cdot$ Remodeling $\cdot$ Topical corticosteroid

\section{Introduction}

Eosinophilic esophagitis (EoE) is a chronic, inflammatory, clinicopathological esophageal condition in children and

\section{Greenhawt}

Division of Allergy and Clinical Immunology and the University of Michigan Food Allergy Center, University of Michigan Medical School, Ann Arbor, MI, USA

\section{S. S. Aceves ( $\square)$}

Division of Allergy, Immunology, Departments of Pediatrics and Medicine, University of California, San Diego, 9500 Gilman Drive, MC-0760, La Jolla, CA 92093-0760, USA

e-mail: saceves@ucsd.edu adults that is hallmarked by symptoms of esophageal dysfunction and acid-independent, multi-level (e.g., distal/ proximal) esophageal eosinophilia of $\geq 15$ eosinophils (eos)/ high-powered field (hpf) on H\&E-stained esophageal biopsies $[1 \bullet, 2 \cdot$. While it is abnormal to have eosinophils within the squamous epithelium of the esophagus, the underlying etiology for esophageal eosinophilia can vary from acid to antigens and combinations thereof. As such, other causes of esophageal eosinophilia should be ruled out including gastroesophageal reflux (GERD), concurrent eosinophilic gastroenteritis disease, systemic eosinophilic disorders, celiac disease, inflammatory bowel disease, infection, vasculitis, drug-induced injury, graft versus host disease, connective tissue disorders, and major motility disorders such as achalasia $[1 \bullet, 2 \bullet, 3]$. It is important, however, to understand that primary EoE may co-exist with at least some of these disorders. For example, treatment of celiac disease with a gluten-free diet will result in concomitant resolution of EoE in $<50 \%$ of cases [4]. Symptoms in EoE are broad, often overlap with other diseases, and can vary based on age. Classic EoE symptoms prominently reflect either esophageal dysfunction (all ages) and/or feeding difficulties (children), with dysphagia being prominent in adults and adolescents [5•]. Endoscopic features include pallor, exudates, linear furrowing, and luminal narrowing, and microscopic histologic findings include eosinophilic micro-abscesses, rete peg elongation, dilated intracellular spaces, degranulated eosinophils, and eosinophil surface layering. However, none are pathognomonic for EoE $[1 \bullet, 5 \bullet]$.

Current epidemiologic evidence indicates an alarming increase in the incidence and prevalence of disease in both the US and abroad $[1 \bullet, 6 \bullet, 7]$. Gene studies and cytokine/ chemokine data strongly implicate that EoE has an allergic etiology and mechanism of disease, involving mast cells, basophils, IL-4, IL-5, IL-13, eotaxin-3, STAT-6, and TSLP 
$[8 \cdot, 9]$. Clinically, as many as $75 \%$ of affected individuals are atopic (or have a family history of atopy), though up to $25 \%$ may be non-atopic [2•]. There is a clear response to allergen removal (between 77 and $98 \%$ in studies), and both food and inhalant allergens have been shown to be triggers of disease $[10-12,13 \cdot 14,15,16 \cdot, 17-19]$.

Treatment options are broad and include dilation, corticosteroids, dietary elimination, and biologic immunomodulation. No treatment strategy has proven superior, and most options have shown efficacy in eliminating symptoms, histologic evidence of disease, or both $[1 \bullet, 2 \bullet, 3,5 \bullet]$. Currently, EoE still lacks a clear best-practice strategy, robust outcome data, or full understanding of the natural history of the disease. In addition, in clinical practice, there is not universal consensus between allergists and gastroenterologists who manage $\operatorname{EoE}[1 \bullet, 2 \bullet, 6 \bullet]$. Moreover, key patient-oriented outcomes, such as quality of life, are just beginning to be investigated [20,21•].

This review will cover the recent developments in EoE in the past year, focusing on updates to the diagnostic guidelines; the emerging evidence for/against protonpump-responsive esophageal eosinophilia (PPI-REE) as a staging classification; updates on dietary avoidance strategies; pathophysiologic updates that may have treatment implications; and potential new methods for esophageal assessment and EoE diagnosis.

\section{Updates on Clinical Guidelines for Diagnosing and Managing EoE}

The American College of Gastroenterology (ACG) recently published clinical guidelines for diagnosing and managing EoE [1•]. Though exclusively authored by gastroenterologists, these guidelines are essentially in line with the 2011 consensus guidelines written by pathologists, allergists, and gastroenterologists [2•], but include the use of the GRADE system for evidence evaluation, which is a strength of the approach [22]. Most recommendations were given a conditional label (trade-offs less certain) and low to moderate evidence ratings (further studies likely or very likely to change the confidence of the recommendation) given limited randomized or controlled supporting data for many scenarios. The main diagnostic recommendation was the need for a trial of 2 months of high-dose proton pump inhibitor therapy in order to rule out gastroesophageal reflux disease (GERD) or proton pump inhibitor-responsive esophageal eosinophilia (PPI-REE) [1•]. In terms of therapeutic options, dietary elimination and topical esophageal corticosteroid remain the mainstays, but the ACG guidelines provide a focus on the evidence surrounding esophageal dilation and advocate a conservative use of dilation to assist with symptomatic treatment from fibrostenotic complications. Although dilation provides symptomatic relief, it does not provide any inflammatory control and is complicated by $75 \%$ of patients experiencing post-dilation pain. Importantly, there is an expansion of the evidence regarding the concept of PPI-REE as a potentially distinct entity from GERD and EoE that also requires clinical follow-up.

\section{The Emergence of PPI-REE as a Key Condition to Rule Out Prior to Diagnosis of EoE}

While esophageal eosinophilia has been noted for decades, this is distinct from the clinicopathologic diagnosis of EoE $[1 \bullet, 2 \bullet]$. All recent guidelines have cautioned against a strict overreliance on an "exact" number of eosinophils that are diagnostic. Indeed, the number of 15 is based not on evidence but on expert opinion. The guidelines all further emphasize that isolated counts $\geq 15$ eos/hpf in asymptomatic patients or patients without a clinical context should not be considered pathognomonic of EoE and that subjects with $<15$ eos/hpf but typical symptoms despite PPI therapy may warrant therapy.

The original consensus guidelines recommended an 8-week trial of high-dose PPI (e.g., twice daily), based on case report evidence that esophageal eosinophilia in some patients may respond to this treatment, and to rule out GERD as a contributing factor [23-27]. It is currently appreciated that there may be at least two distinct populations of patients with esophageal eosinophilia, which has prompted a shifting of the nomenclature-those with abnormal $\mathrm{pH}$ studies and GERD with high numbers of eosinophils and those with normal $\mathrm{pH}$ and eosinophilia responsive to PPI [2•]. For this reason, the new term PPIREE more accurately describes the symptomatic patient with high-grade and often diffuse esophageal eosinophilia who responds clinically and histologically to the 8-week high-dose PPI. Future studies will need to decipher whether individuals with PPI-REE have antigen-induced eosinophilia and what mediates the therapeutic effect of PPI. To this end, recent work has demonstrated the PPI effects may not merely be due to acid suppression but also to decreasing pro-eosinophil chemokines. Using esophageal EoE epithelial cells, Zheng et al. demonstrated that omeprazole decreases eotaxin-3 induction by IL-13, supporting the hypothesis that PPIs may be able to inhibit eosinophils via a gastric-acid independent, immunologically mediated pathway. Omaprazole at $5 \mu \mathrm{M}$ or higher concentrations decreased IL-4-stimulated eotaxin-3 protein secretion and mRNA expression, likely through chromatin remodeling at the promoter, but had no effect on eotaxin-3 mRNA stability, STAT-6 phosphorylation or nuclear translocation. 
Clinically, the evidence supporting PPI-REE remains largely based on uncontrolled, descriptive studies, although some randomized trial data have emerged $[1 \bullet, 2 \bullet, 3,28 \cdot]$. Schroeder et al. [29] performed a retrospective analysis of 204 children diagnosed with EoE to identify responders to an 8-week PPI treatment and differentiate responders from non-responders. Seven patients with clinico-pathologic response were identified, two of whom re-developed esophageal eosinophilia within 1.5-2 years of treatment, and there were no clinical indicators to differentiate transient from long-term PPI responders. More importantly, $77 \%$ of children showed no PPI response. The data from Schroeder et al. [29] emphasize the importance of the PPI trial, but also underscore that these patients need long-term follow-up given disease overlap and unclear differentiating factors between EoE and PPI-REE. These findings closely mirror work by Dohil, Newbury, and Aceves, who described transient response followed by recrudescence of esophageal eosinophilia in four children treated with (and remaining on) PPI, independent of seasonal variation. Dohil, Newbury, and Aceves caution against a quick diagnosis of PPI-REE, urge long-term follow-up of this approach, and caution reliance upon isolated acid reduction as the optimal long-term treatment strategy in this subset of subjects [27].

Adult studies have also supported the presence of PPIREE. Moawad et al. [30] performed a randomized, controlled, single-blinded trial in 42 adult patients treated with topical fluticasone proprionate versus esomeprazole and found no significant differences between the response rates to topical esophageal steroids or PPI. Symptom scores on the dysphagia questionnaire were significantly improved in the PPI-treated group, but this improvement was not related to the overall eosinophil count change, underscoring the disconnect that commonly occurs between symptoms and inflammation.

Dellon et al. conducted a large prospective study of an 8-week PPI trial in adults undergoing EGD, stratified by dysphagia ( $n=173$ with dysphagia and $n=50$ without). Subjects with $\geq 15$ eos/hpf on biopsy received a 2-month high-dose PPI trial (66/173 with dysphagia, 2/50 without dysphagia), followed by re-biopsy. Response was noted only in 24/66 total subjects with dysphagia, and these patients were indistinguishable by symptoms or endoscopic features from 40 subjects not responding to the PPI trial who were diagnosed with EoE [31•]. Vazquez-Elizondo et al. conducted an open label trial of an 8-week 20-mg twice-daily esomeprazole trial in 60 consecutive adult patients who had esophageal eosinophilia $>15$ eos/hpf. Using a remission cutoff of $<5$ eos/hpf and an improvement cutoff of 5-14 eos/hpf, $25 \%$ achieved full remission, $56.6 \%$ improved, and $71.6 \%$ had symptomatic response. Decreases in eosinophil counts were noted more frequently (non-significantly) in those with more prominent distal than proximal eosinophilia, and counts were significantly lower in those with evidence of GERD (e.g., heartburn/ regurgitation by history). Molina-Infante et al. summarized the results of ten studies investigating PPI response in patients with suspected EoE, encompassing 152 children and 106 adults. Overall there was a symptom response to PPI in $78-86 \%$ of children and $25-80 \%$ of adults, and noted histologic remission in $23-40 \%$ of children and $33-61 \%$ of adults. Overall $50-85 \%$ of subjects had symptom improvement without histologic remission, and 6 patients had relapse despite ongoing PPI therapy. PPI-REE was diagnosed with higher frequency in patients with $\mathrm{pH}$ probe-proven GERD [32].

\section{New Insights into Dietary Therapy: Flexible Options Exist Allowing Tailoring}

EoE is a chronic, antigen-driven disease that responds to dietary elimination. Evidence supporting a role for food antigen avoidance in treating EoE dates back to initial work by Kelly et al. and furthered by work from Markowitz et al. and Liacouras et al., demonstrating high rates of symptomatic response and histologic normalization with use of an exclusively elemental diet [10, 12,33]. Targeted dietary elimination, first described by Spergel et al., identified the 13 most common pediatric food triggers through skin testing (PST) and atopy patch testing (APT), and provides an alternative that allows more selective exclusion of foods with nearly $80 \%$ success in terms of symptomatic and histologic normalization [13•, 34-36]. However, other groups have described difficulty in replicating these data, highlighting food tests (milk, wheat) with poor reliability, as well as frustration regarding high variability in the APT process $[2 \bullet, 16 \bullet]$. A third strategy, empiric elimination, continues to grow in popularity. Kagalwala et al. showed that eliminating the six most common allergenic food groups in the US (6-food elimination diet [SFED], consisting of milk, egg, soy, wheat, peanut/tree nuts, and seafood), without allergy testing, resulted in similar rates of symptomatic and histologic remission to an elemental diet control group (74\% response for SFED versus $88 \%$ response in the elemental control group) [14, 37].

In the last year, two large retrospective case series from prominent university referral centers were published, attempting to contrast efficacy of the three main dietary strategies. Henderson et al. [16•] compared remission rates among three cohorts of patients undergoing an elemental diet, classic SFED, or tailored elimination diet in 98 pediatric patients on PPI therapy. All three diets showed successful peak eosinophil count reduction. The elemental diet produced a peak count reduction from 51 to 1 eos/hpf, SFED from 76.5 to $2.5 \mathrm{eos} / \mathrm{hpf}$, and tailored diet from 38 to 
Table 1 Comparison of benefits and detriments of the three dietary management styles

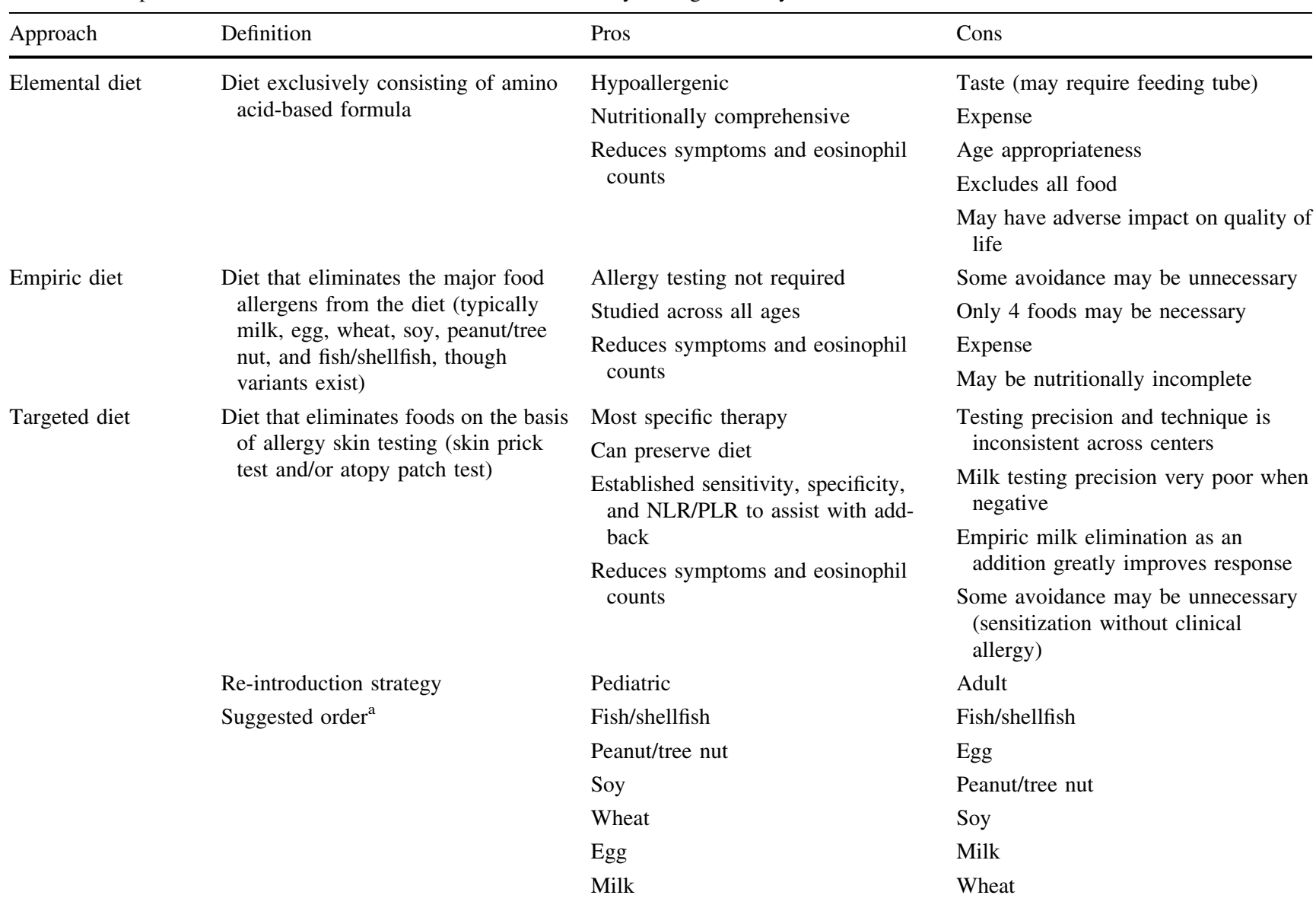

$N L R / P L R$ negative/positive likelihood ratio

${ }^{a}$ Data are best established for classic SFED but may be applicable to both elemental and targeted diets

7 eos/hpf. There were no significant proportional differences in the number of patients achieving remission $(<15$ eos/hpf). There were significant differences in the odds of remission between elemental and SFED, and elemental and tailored elimination therapy, but not between the tailored and the SFED diet. Henderson et al. [16•] noted poor NPVs for the four most commonly introduced foods (milk, egg, soy, and wheat), ranging from 40 to $67 \%$ based on PST and APT. However, it clearly demonstrates the potential for multiple successful dietary management strategies.

In a similar study, Spergel et al. reported retrospective data on 7 dietary styles in 941 pediatric EoE patients evaluated between 2000 and 2011 [14]. The diets evaluated included empiric milk avoidance; empiric milk, egg, and wheat avoidance; classic SFED; tailored elimination; tailored elimination plus empiric milk elimination; empiric milk, egg, wheat, soy, and meat avoidance; and a vegan diet. A causative food, defined as histologic disease recurrence on food re-introduction, was identified in 319 patients, most commonly milk, followed by egg, wheat, beef, soy, and chicken. Remission rates $(<1$ eos/hpf) were nearly identical for both targeted elimination and SFED (53\%), $47 \%$ for empiric milk/egg/wheat, and $30 \%$ with just empiric milk elimination alone. The highest observed remission rates were with targeted elimination combined with an empiric milk elimination and empiric milk/egg/ wheat/soy/meat elimination diet, which both had a $77 \%$ response rate. Together these studies confirm that there is potential for multiple successful dietary management strategies that include allergy testing-based diets in children $\left[13 \bullet, 16^{\bullet}\right.$. Table 1 reviews the pros and cons of the three major dietary strategies.

On the other hand, dietary studies that have focused exclusively on adult patients have provided conflicting data as to the efficacy of allergen testing-based diets. Gonsalves et al. conducted a prospective, non-randomized, uncontrolled study of an SFED in 50 adult EoE patients, noting $94 \%$ had symptom improvement, $74 \%$ responded, and $64 \%$ achieved remission [15]. However, isolated PSTbased allergy testing predicted a trigger in just $13 \%$ [15]. In a 6-week, prospective, uncontrolled study of 67 adults, 
Lucendo et al. conducted a prospective 6-week SFED variant (avoidance of cereal grains, dairy, egg, seafood, legumes, nut/seed, and soy) and noted $75.5 \%$ achieving counts $<5 / \mathrm{hpf}$, but this was poorly concordant with allergy testing results. However, 15 of the 49 patients who maintained the diet for 2 years remained in histologic remission [38]. Lastly, Molina-Infante et al. noted minimal effect of a 6-week testing based targeted elimination diet in an uncontrolled, non-randomized adult cohort of 22 patients, noting 10/15 had no histologic change [39]. It remains unclear whether a particular dietary approach is more effective in certain age ranges, and additional adult studies are warranted.

One other study of interest deals with the concept of epitope specificity in the recognition of food allergen triggers in EoE. In IgE-mediated food allergy certain major food epitopes are conformational and therefore labile with extensive heat and tolerated by many allergic individuals who have immediate hypersensitivity to food antigens [40]. Specifically this has been identified with both cow's milk and hen's egg, and between 70 and $80 \%$ of milk/egg allergic individuals may tolerate "baked" forms of these product (e.g., extensively heated for $>30 \mathrm{~min}$ at a temperature $>350^{\circ} \mathrm{F}$ ), which also may be associated with phenotype of faster overall allergy resolution [41]. It has been unclear whether these foods are safe for individuals with milk-triggered EoE. In a case series of 15 patients with confirmed milk-triggered EoE, Leung et al. showed that $11 / 15$ patients remained in remission after 6 weeks of baked milk ingestion [42•].

\section{New Pathophysioloigic Insights Have Potential Therapeutic Value}

In 2010, genome-wide array screening of children with EoE identified a gain of function mutation associated with a loci of interest on gene 5q22, encoding thymic stromal lymphopoetin (TSLP), a master Th2 cytokine essential for allergic development [43]. However it was unclear exactly how this mediated disease. Recently, study by Noti et al. [8•] discovered that TSLP and basophils are crucial mediators in an IgE-independent experimental murine model of EoE, which may indicate a new potential therapeutic target.

In a murine model established through epicutaneous food sensitization on an atopic dermatitis-like skin lesion, mice developed EoE-like disease with expansion of systemic basophil production and a trend towards higher TSLP expression compared to control mice. TSLP-deficient mice treated with a TSLP-blocking antibody were protected from experimental EoE, as were mice treated with basophil depletion. Importantly, the mice in this model system developed food impactions, the rates of which were significantly diminished when TSLP or basophils were decreased. These investigators further established $\mathrm{IgE}$ independence of $\mathrm{EoE}$ in this system. In translational studies, biopsy samples with EoE showed higher immunohistochemical staining of TSLP or basophil markers, and the basophil staining correlated with increasing eosinophil counts; peripheral blood mononuclear cells from subjects with active or inactive EoE that expressed the gain-of-function TSLP polymorphism were noted to have higher numbers of basophils [8•].

Other murine model systems demonstrated that eosinophils are important for esophageal strictures, fibrosis, and certain histological features such as basal zone hyperplasia. In addition, interleukins such as IL-13 were demonstrated to cause strictures that were independent of eosinophilia $[44,45]$. However, other functional and histologic features such as those dependent on esophageal smooth muscle appear to be independent of eosinophils. Both increased smooth muscle contraction and decreased smooth muscle relaxation were independent of eosinophilia, and smooth muscle hypertrophy and hyperplasia relied on esophageal mastocytosis [45, 46]. With regard to fibrosis, CCL18 has been found to be elevated in EoE [47]. Studies by the Luster laboratory identified CCR8 as the CCL18 receptor, and both were found to be elevated in active EoE biopsies [48]. Together, these studies have translational implications for both anti-eosinophil strategies using biologics that provide siglec-8, IL-13, CCR8, and/or mast cell blockade.

Data for anti-IL-5 as treatment for EoE have been largely disappointing in controlled trials $[1 \bullet, 2 \bullet]$. However, investigation has focused on the eosinophil response. Both Abonia et al. [50] and Aceves et al. [49] have indicated that the mast cell, and not the eosinophil, may play a more important role in EoE. Mast cells outnumber eosinophils in the smooth muscle of affected esophageal tissue, and tryptase-secreting mast cells can associate with dysphagia and greater levels of TGF $\beta 1$ (which may mediate esophageal smooth muscle contractility) [49]. Using nested biopsy samples from a prior anti-IL-5 clinical trial, Otani et al. [51•] noted that mast cell numbers decreased in $77 \%$ of anti-IL-5 treated subjects, which correlated to decreased eosinophil counts, and that responders had significantly larger mast cell decreases than non-responders. IL-9, a key mast cell growth factor, was noted to be produced from the esophageal eosinophils, and was also decreased after treatment [51•]. It is potentially notable that while eosinophil decreases did not correlate with symptom changes, mast cell decreases did associate with symptom response in this study.

Krishnamurthy et al., building on data that PARP14 acts as a transcription cofactor for STAT-6 (critical for eotaxin3 expression), found PARP14 and PARP1 gene expression was upregulated in esophageal biopsies of children with 
EoE versus controls, and PARP14 RNA was also upregulated [52]. Using biopsies from two clinical populations, PARP14 and eotaxin-3 expressions were strongly correlated. Transfecting an esophageal cell line with PARP14 in the presence of STAT-6 increased eotaxin-3 promoter activity, and IL-4 and IL-13-induced expression of eotaxin3 mRNA was attenuated by PARP inhibition [52]. This is preliminary work, but may be a future pathway for therapeutic exploitation.

\section{Update on a New Diagnostic Modalities and Natural History}

Diagnosing and monitoring of EoE are heavily dependent on obtaining an esophageal biopsy. Non-invasive methods based on symptom algorithms are poorly predictive eosinophil counts $[1 \bullet 2 \bullet]$. Furuta et al. [53•] recently described an effective, minimally invasive monitoring technique. Using a modified capsule and 90-cm string (Enterotest, Pilpitas, CA), patients swallowed a capsule, and the proximal end of the string was used to assess esophageal luminal secretions for the presence of eosinophil secondary granule proteins ([ESGP] MBP-1, EPX, EDN, ECP, and CLC/Gal-10). Children with active EoE, EoE in remission, GERD, and with no esophageal disease swallowed the capsule/string and were monitored for $24 \mathrm{~h}$ followed by string removal, upper endoscopy with biopsy, and analysis of the biopsy and string for ESGPs. ESGP levels from both the string and the mucosal biopsy were able to differentiate those with active EoE from those in remission, with GERD, or with no esophageal disease, and there was significantly strong correlation between biopsy and ESGP findings. Indeed, statistical analysis revealed high receiver-operator characteristic scores, an indicator of accuracy and precision, for the ability of the string test to predict EoE [53•]. Though this technique requires further study, it is an exceptionally promising diagnostic modality.

Similarly, an adult study demonstrated that the use of a test that quantifies esophageal compliance, endoFLIP, predicted the risk of future food impaction based on esophageal rigidity [54•]. Although not yet reported in children, this test may provide a powerful tool for the assessment of esophageal function and remodeling. Indeed, esophageal tissue remodeling with resultant fibrosis, angiogenesis, and smooth muscle hypertrophy appears to underlie the major EoE complications of food impactions and stricture as well as the predominant EoE symptom of dysphagia [55-57]. Underscoring the importance of tissue remodeling, pivotal adult studies have demonstrated that long-standing, untreated EoE progresses almost uniformly to esophageal stricture [58•, 59•]. These studies have important implications for pediatric practitioners since the hope is that the control of inflammation and remodeling will translate into the control of potential stricture formation.

Lastly, 2013 also saw the first implementation of molecular diagnostics to EoE with the use of the EoE diagnostic panel (EDP), a fluidic gene microarray applicable to fresh and paraffin-embedded esophageal biopsy specimens. The EDP demonstrated $98 \%$ specificity and $96 \%$ sensitivity for distinguishing a diagnosis of EoE in children. This molecular tool described by the Rothenberg laboratory could have a significant impact on EoE and, if combined with less invasive methods, could revolutionize the field of EoE diagnostics [60•].

\section{Conclusion}

There continue to be breakthroughs in the understanding of the underlying pathophysiology of EoE, how to diagnose EoE, and how to most effectively treat EoE. Significant progress has been made in the past 12-15 months. However, many barriers remain, including poor differentiation of EoE from other diseases with esophageal eosinophilia, reliance on biopsy for diagnosis, unclear superiority of several treatment approaches, and lack of an effective biologic treatment. Future work aimed at addressing these issues will undoubtedly have important implications for our understanding and care of EoE.

Disclosure Matthew Greenhawt has consulted for Nutricia, Deerfield Industries, Franklin Group, and the National Peanut Board (educational advisory council); has board membership with Nutricia; has received payment for lectures and development of educational presentations from Nutricia. Seema S. Aceves reports grants from NIH/NIAID and from APFED/AAAAI ART, outside the submitted work. In addition, Dr. Aceves has a patent oral viscous budesonide issued to UCSD, for which Dr. Aceves is a co-inventor.

Human and Animal Rights and Informed Consent This article does not contain any studies with human or animal subjects performed by any of the authors.

\section{References}

Papers of particular interest, published recently, have been highlighted as:

- Of importance

1. - Dellon ES, Gonsalves N, Hirano I, Furuta GT, Liacouras CA, Katzka DA. ACG clinical guideline: evidence based approach to the diagnosis and management of esophageal eosinophilia and eosinophilic esophagitis (EoE). Am J Gastroenterol. 2013;108:679-92; quiz 93. These are the most recent diagnostic and management criteria on EoE, authored exclusively by 
gastroenterologists, using the GRADE system. A heavy emphasis is placed on the importance of differentiating PPI-responsive esophageal eosinophilia from EoE.

2. - Liacouras CA, Furuta GT, Hirano I, et al. Eosinophilic esophagitis: updated consensus recommendations for children and adults. J Allergy Clin Immunol. 2011;128:3-20 e6; quiz 1-2. These are the most updated consensus Allergy and Gastroenterology guidelines on the diagnosis and management of EoE.

3. Furuta GT, Liacouras CA, Collins MH, et al. Eosinophilic esophagitis in children and adults: a systematic review and consensus recommendations for diagnosis and treatment. Gastroenterology. 2007;133:1342-63.

4. Leslie C, Mews C, Charles A, Ravikumara M. Celiac disease and eosinophilic esophagitis: a true association. J Pediatr Gastroenterol Nutr. 2010;50:397-9.

5. - Greenhawt M, Aceves SS, Spergel JM, Rothenberg ME. The management of eosinophilic esophagitis. J Allergy Clin Immunol. 2013;1:332-40. A recent review evaluating dietary therapy options in treating EoE. It includes a thorough discussion of the pros and cons of each style, and provides guidelines obtained from current evidence on food re-introduction in EoE.

6. - Dellon ES, Jensen ET, Martin CF, Shaheen NJ, Kappelman MD. Prevalence of eosinophilic esophagitis in the United States. Clin Gastroenterol Hepatol. 2013. This is a key study providing the first population-based estimates of the prevalence of EoE in the US in both children and adults.

7. van Rhijn BD, Verheij J, Smout AJ, Bredenoord AJ. Rapidly increasing incidence of eosinophilic esophagitis in a large cohort. Neurogastroenterol Motil. 2013;25(47-52):e5.

8. - Noti M, Wojno ED, Kim BS, et al. Thymic stromal lymphopoietin-elicited basophil responses promote eosinophilic esophagitis. Nat Med. 2013;19:1005-13. This is a very important study that elucidates the role of the basophil in an IgE-independent model of EoE and further clarifies the potential role of TSLP as a key cytokine involved in pathogenesis of EoE.

9. Rothenberg ME. Biology and treatment of eosinophilic esophagitis. Gastroenterology. 2009;137:1238-49.

10. Kelly KJ, Lazenby AJ, Rowe PC, Yardley JH, Perman JA, Sampson HA. Eosinophilic esophagitis attributed to gastroesophageal reflux: improvement with an amino acid-based formula. Gastroenterology. 1995;109:1503-12.

11. Liacouras CA, Wenner WJ, Brown K, Ruchelli E. Primary eosinophilic esophagitis in children: successful treatment with oral corticosteroids. J Pediatr Gastroenterol Nutr. 1998;26:380-5.

12. Markowitz JE, Spergel JM, Ruchelli E, Liacouras CA. Elemental diet is an effective treatment for eosinophilic esophagitis in children and adolescents. Am J Gastroenterol. 2003;98:777-82.

13. - Spergel JM, Brown-Whitehorn TF, Cianferoni A, et al. Identification of causative foods in children with eosinophilic esophagitis treated with an elimination diet. J Allergy Clin Immunol. 2012. This is a large cohrt study evaluating the effectiveness of several different food elimination diets. As well, it provides an update to the precision of food allergen diagnostic testing in EoE.

14. Kagalwalla AF, Sentongo TA, Ritz S, et al. Effect of six-food elimination diet on clinical and histologic outcomes in eosinophilic esophagitis. Clin Gastroenterol Hepatol. 2006;4:1097-102.

15. Gonsalves N, Yang GÄ, Doerfler B, Ritz S, Ditto AM, Hirano I. Elimination diet effectively treats eosinophilic esophagitis in adults: food reintroduction identifies causative factors. Gastroenterology. 2012;142(1451-9):e1.

16. - Henderson CJ, Abonia JP, King EC, et al. Comparative dietary therapy effectiveness in remission of pediatric eosinophilic esophagitis. J Allergy Clin Immunol 2012;129:1570-8. This is a retrospective cohort study comparing the effects of the three major dietary elimination strageties on the rates of achieving remission in EoE. It noted superior effects for elemental diet, though patients were not randomized to any diet, and some patients in each of the three diet groups achieved remission.

17. Simon D, Marti H, Heer P, Simon HU, Braathen LR, Straumann A. Eosinophilic esophagitis is frequently associated with IgEmediated allergic airway diseases. J Allergy Clin Immunol. 2005;115:1090-2.

18. Plaza-Martin AM, Jimenez-Feijoo R, Andaluz C, et al. Polysensitization to aeroallergens and food in eosinophilic esophagitis in a pediatric population. Allergol Immunopathol (Madr). 2007;35:35-7.

19. Simon D, Straumann A, Dahinden C, Simon HU. Frequent sensitization to Candida albicans and profilins in adult eosinophilic esophagitis. Allergy. 2013;68:945-8.

20. Franciosi JP, Hommel KA, DeBrosse CW, et al. Quality of life in paediatric eosinophilic oesophagitis: what is important to patients? Child Care Health Dev. 2012;38:477-83.

21. - Franciosi JP, Hommel KA, Bendo CB, et al. PedsQL eosinophilic esophagitis module: feasibility, reliability and validity. J Pediatr Gastroenterol Nutr 2013. This is a study that describes the formation and validation of the first quality of life measure (parental proxy report of the child's quality of life) in EoE.

22. Kavanagh BP. The GRADE system for rating clinical guidelines. PLoS Med. 2009;6:e1000094.

23. Ngo P, Furuta GT, Antonioli DA, Fox VL. Eosinophils in the esophagus-peptic or allergic eosinophilic esophagitis? Case series of three patients with esophageal eosinophilia. Am J Gastroenterol. 2006;101:1666-70.

24. Molina-Infante J, Ferrando-Lamana L, Fernandez-Bermejo M, Porcel-Carreno S. Eosinophilic esophagitis in GERD patients: a clinicopathological diagnosis using proton pump inhibitors. Am J Gastroenterol. 2009;104:2856-7.

25. Sayej WN, Patel R, Baker RD, Tron E, Baker SS. Treatment with high-dose proton pump inhibitors helps distinguish eosinophilic esophagitis from noneosinophilic esophagitis. J Pediatr Gastroenterol Nutr. 2009;49:393-9.

26. Molina-Infante J, Ferrando-Lamana L, Ripoll C, et al. Esophageal eosinophilic infiltration responds to proton pump inhibition in most adults. Clin Gastroenterol Hepatol. 2011;9:110-7.

27. Dohil R, Newbury RO, Aceves S. Transient PPI responsive esophageal eosinophilia may be a clinical sub-phenotype of pediatric eosinophilic esophagitis. Dig Dis Sci. 2012;57:1413-9.

28. - Zhang X, Cheng E, Huo X, et al. Omeprazole blocks STAT6 binding to the eotaxin-3 promoter in eosinophilic esophagitis cells. PLoS ONE 2012;7:e50037. A key paper describing evidence of how PPI may act as an anti-inflammatory agent that inhibits eosinophils through a mechanism independent of gastric acid suppression.

29. Schroeder S, Capocelli KE, Masterson JC, et al. Effect of proton pump inhibitor on esophageal eosinophilia. J Pediatr Gastroenterol Nutr. 2013;56:166-72.

30. Moawad FJ, Veerappan GR, Dias JA, Baker TP, Maydonovitch CL, Wong RK. Randomized controlled trial comparing aerosolized swallowed fluticasone to esomeprazole for esophageal eosinophilia. Am J Gastroenterol. 2013;108:366-72.

31. - Dellon ES, Speck O, Woodward K, et al. Clinical and endoscopic characteristics do not reliably differentiate PPI-responsive esophageal eosinophilia and eosinophilic esophagitis in patients undergoing upper endoscopy: a prospective cohort study. Am J Gastroenterol 2013;108:1854-60. This is a large cohort study that demonstrates PPI-REE and EoE cannot be diferentiated from one another by symptoms or endoscopic/histologic features.

32. Molina-Infante J, Katzka DA, Gisbert JP. Review article: proton pump inhibitor therapy for suspected eosinophilic oesophagitis. Aliment Pharmacol Ther. 2013;37:1157-64.

33. Liacouras CA, Spergel JM, Ruchelli E, et al. Eosinophilic esophagitis: a 10-year experience in 381 children. Clin Gastroenterol Hepatol. 2005;3:1198-206. 
34. Spergel JM, Beausoleil JL, Mascarenhas M, Liacouras CA. The use of skin prick tests and patch tests to identify causative foods in eosinophilic esophagitis. J Allergy Clin Immunol. 2002;109: 363-8.

35. Spergel JM, Andrews T, Brown-Whitehorn TF, Beausoleil JL, Liacouras CA. Treatment of eosinophilic esophagitis with specific food elimination diet directed by a combination of skin prick and patch tests. Ann Allergy Asthma Immunol. 2005;95:336-43.

36. Spergel JM, Brown-Whitehorn T, Beausoleil JL, Shuker M, Liacouras CA. Predictive values for skin prick test and atopy patch test for eosinophilic esophagitis. J Allergy Clin Immunol. 2007;119:509-11.

37. Kagalwalla AF, Shah A, Li BUK, et al. Identification of specific foods responsible for inflammation in children with eosinophilic esophagitis successfully treated with empiric elimination diet. J Pediatr Gastroenterol Nutr. 2011;53:145-9. doi:10.1097/MPG. 0b013e31821cf503.

38. Lucendo AJ, Arias A, Gonzalez-Cervera J, et al. Empiric 6-food elimination diet induced and maintained prolonged remission in patients with adult eosinophilic esophagitis: a prospective study on the food cause of the disease. J Allergy Clin Immunol. 2013;131:797-804.

39. Molina-Infante J, Martin-Noguerol E, Alvarado-Arenas M, Porcel-Carreno SL, Jimenez-Timon S, Hernandez-Arbeiza FJ. Selective elimination diet based on skin testing has suboptimal efficacy for adult eosinophilic esophagitis. J Allergy Clin Immunol. 2012;130:1200-2.

40. Boyce JA, Assa'ad A, Burks AW, et al. Guidelines for the diagnosis and management of food allergy in the United States: report of the NIAID-sponsored expert panel. J Allergy Clin Immunol. 2010;126:S1-58.

41. Konstantinou GN, Kim JS. Paradigm shift in the management of milk and egg allergy: baked milk and egg diet. Immunol Allergy Clin North Am. 2012;32:151-64.

42. - Leung J, Hundal NV, Katz AJ, et al. Tolerance of baked milk in patients with cow's milk-mediated eosinophilic esophagitis. J Allergy Clin Immunol 2013;132:1215-6 e1. Provides insightful pilot-level study into the concept of epitope specificity in dietary management of EoE, in that heal-labile milk epitopes may be well-tolerated by milk sensitive children with EoE.

43. Rothenberg ME, Spergel JM, Sherrill JD, et al. Common variants at 5 q22 associate with pediatric eosinophilic esophagitis. Nat Genet. 2010;42:289-91.

44. Mavi P, Rajavelu P, Rayapudi M, Paul RJ, Mishra A. Esophageal functional impairments in experimental eosinophilic esophagitis. Am J Physiol Gastrointest Liver Physiol. 2012;302:G1347-55.

45. Niranjan R, Rayapudi M, Mishra A, Dutt P, Dynda S. Pathogenesis of allergen-induced eosinophilic esophagitis is independent of interleukin (IL)-13. Immunol Cell Biol. 2013;91:408-15.

46. Niranjan R, Mavi P, Rayapudi M, Dynda S, Mishra A. Pathogenic role of mast cells in experimental eosinophilic esophagitis. Am J Physiol Gastrointest Liver Physiol. 2013;304:G1087-94.

47. Lucendo AJ, Arias A, De Rezende LC, et al. Subepithelial collagen deposition, profibrogenic cytokine gene expression, and changes after prolonged fluticasone propionate treatment in adult eosinophilic esophagitis: a prospective study. J Allergy Clin Immunol. 2011;128:1037-46.
48. Islam SA, Ling MF, Leung J, Shreffler WG, Luster AD. Identification of human CCR8 as a CCL18 receptor. J Exp Med. 2013;210:1889-98.

49. Aceves SS, Chen D, Newbury RO, Dohil R, Bastian JF, Broide DH. Mast cells infiltrate the esophageal smooth muscle in patients with eosinophilic esophagitis, express TGF-beta1, and increase esophageal smooth muscle contraction. J Allergy Clin Immunol. 2010;126(1198-204):e4.

50. Abonia JP, Blanchard C, Butz BB, et al. Involvement of mast cells in eosinophilic esophagitis. J Allergy Clin Immunol. 2010;126:140-9.

51. - Otani IM, Anilkumar AA, Newbury RO, et al. Anti-IL-5 therapy reduces mast cell and IL-9 cell numbers in pediatric patients with eosinophilic esophagitis. J Allergy Clin Immunol 2013;131: 1576-82. An important study reprsenting post hoc analysis of samples from an earlier anti-IL-5 study that showed that anti-IL5 decreased mast cell numbers in treated tissue, and decreased esophageal eosinophil derived IL-9.

52. Krishnamurthy P, Sherrill JD, Parashette K, et al. Correlation of increased PARP14 and CCL26 expression in biopsies from children with eosinophilic esophagitis. J Allergy Clin Immunol. 2013;133(2):577-80.

53. • Furuta GT, Kagalwalla AF, Lee JJ, et al. The oesophageal string test: a novel, minimally invasive method measures mucosal inflammation in eosinophilic oesophagitis. Gut 2013;62: 1395-405. A very novel study that describes a minimally invasive procedure for diagnosing EoE.

54. • Nicodeme F, Hirano I, Chen J, et al. Esophageal distensibility as a measure of disease severity in patients with eosinophilic esophagitis. Clin Gastroenterol Hepatol 2013;11:1101-7 e1. A very novel study that explores a method of assessing esophageal wall thickness as a marker of fibrosis in EoE.

55. Mishra A, Wang M, Pemmaraju VR, et al. Esophageal remodeling develops as a consequence of tissue specific IL-5-induced eosinophilia. Gastroenterology. 2008;134:204-14.

56. Straumann A, Conus S, Degen L, et al. Budesonide is effective in adolescent and adult patients with active eosinophilic esophagitis. Gastroenterology 2010;139:1526-37, 37e1.

57. Aceves SS, Newbury RO, Dohil R, Bastian JF, Broide DH. Esophageal remodeling in pediatric eosinophilic esophagitis. J Allergy Clin Immunol. 2007;119:206-12.

58. - Schoepfer AM, Safroneeva E, Bussmann C, et al. Delay in diagnosis of eosinophilic esophagitis increases risk for stricture formation in a time-dependent manner. Gastroenterology 2013;145:1230-6 e1-2. One of the first long-term outcome studies in EoE demonstrating that delay in diagnosis and treatment of EoE is associated with some risk of stricture formation.

59. - Dellon ES, Kim HP, Sperry SL, Rybnicek DA, Woosley JT, Shaheen NJ. A phenotypic analysis shows that eosinophilic esophagitis is a progressive fibrostenotic disease. Gastrointest Endosc 2013. One of the first studies to identify different phenotypes of disease in EoE.

60. - Wen T, Stucke EM, Grotjan TM, et al. Molecular diagnosis of eosinophilic esophagitis by gene expression profiling. Gastroenterology 2013;145:1289-99. This is an important paper describing a novel diagnostic technique for investigating genetic and molecular markers through parafin stained esophageal biopsy samples. 